Supplement of

\title{
Quantification and evaluation of atmospheric ammonia emissions with different methods: a case study for the Yangtze River Delta region, China
}

Yu Zhao et al.

Correspondence to: Yu Zhao (yuzhao@nju.edu.cn)

The copyright of individual parts of the supplement might differ from the CC BY 4.0 License. 


\section{Table list}

Table S1. The annual numbers of livestock and poultry by prefectural city in YRD (10 000)

Table S2. The fertilizer consumption by type and prefectural city in the YRD region, and the provincial-level total consumption from statistics (metric tons).

Table S3. The $\mathrm{NH}_{3}$ emission factors used in E1.

Table S4. The relevant parameters used for correction of emission factors of fertilizer activity in E2.

Table S5. The parameters used in estimates of annual TAN excretion per cattle.

Table S6. The temperature-dependant emission factors by stage/phase in livestock farming in E2.

Table S7. Model performance for meteorological parameters in D2.

Table S8. The inter-annual change in $\mathrm{SO}_{2}$ and $\mathrm{NO}_{2}$ VCDs for the YRD region 2012-2014 (\%)

\section{Figure list}

Figure S1. The corrected $\mathrm{NH}_{3}$ volatilization of urea and ABC application in E2.

Figure S2. Total ammoniacal nitrogen (TAN) for three main raising systems (taken from Huang et al., 2012).

Figure S3. The daily $\mathrm{NH}_{3}$ concentration at JS-PAES for October 2014 from observation and simulation with E1 and E2.

Figure S4. The $\mathrm{NH}_{3}$ volatilization rates under different soil $\mathrm{pH}$ values for urea (a) and ABC fertilizer (b). The blue dots indicate the values by grid in the YRD region in E2, while the red dots indicate the results from the field measurements by Zhong et al. (2006) (a) and Zhang et al. (2002) (b). The solid lines and equations were obtained from linear regression for the gridded values in the YRD region in E2. 


\section{Tables}

Table S1. The annual numbers of livestock and poultry by prefectural city in YRD (10 000)

\begin{tabular}{|c|c|c|c|c|c|c|c|c|c|c|c|c|c|c|c|c|c|}
\hline & Beef & Cow & Sow & Hog & Goat & Sheep & Layer & Laying Duck & Broiler & Meat duck & Goose & Horse & Donkey & Mule & Rabbit & Cattle & Buffalo \\
\hline Shanghai & 0.10 & 5.80 & 13.90 & 243.13 & 26.70 & 1.40 & 379.05 & 37.61 & 23.81 & 1051.68 & 140.00 & - & - & - & 9.60 & - & - \\
\hline Nanjing & - & 1.75 & - & - & 11.73 & - & 491.68 & 119.51 & 12.57 & 562.96 & 108.92 & - & - & - & 17.49 & 0.01 & 1.08 \\
\hline Xuzhou & - & - & - & - & 218.91 & 8.63 & 3783.84 & 919.73 & 96.01 & 4301.53 & 832.24 & 0.13 & 1.59 & 0.87 & - & - & - \\
\hline Changzhou & 0.01 & 0.54 & 49.10 & 83.62 & 6.05 & 0.14 & 166.90 & 40.57 & 18.98 & 850.37 & 164.53 & - & - & - & - & - & - \\
\hline Suzhou & - & 2.39 & 76.69 & 103.46 & 4.36 & 6.37 & 278.52 & 67.70 & 9.82 & 440.12 & 85.15 & - & - & - & 9.32 & - & - \\
\hline Nantong & - & 0.75 & 270.76 & 394.71 & 227.33 & - & 2984.22 & 725.37 & 47.46 & 2126.22 & 411.37 & - & - & - & - & - & 0.32 \\
\hline Lianyungang & 10.89 & 6.36 & 20.03 & 300.50 & 18.04 & - & 612.70 & 148.93 & 13.86 & 620.76 & 120.10 & - & 0.49 & - & - & - & - \\
\hline Huai'an & 2.21 & 0.82 & 15.68 & 267.11 & 23.29 & - & 849.60 & 206.51 & 29.18 & 1307.15 & 252.90 & - & - & - & - & - & 3.06 \\
\hline Yancheng & - & 1.97 & 41.79 & 735.62 & 138.68 & - & 7591.19 & 486.72 & 81.09 & 3633.09 & 702.91 & - & 0.02 & - & 228.00 & 1.38 & 1.10 \\
\hline Yangzhou & 0.41 & 0.47 & 5.12 & 135.72 & 6.26 & - & 651.50 & 391.05 & 19.44 & 870.89 & 168.49 & - & - & - & 11.09 & - & - \\
\hline Zhenjiang & - & - & 40.58 & 42.30 & 4.17 & - & 164.29 & 39.93 & 8.07 & 361.71 & 69.98 & - & - & - & - & - & - \\
\hline Suqian & 5.92 & 7.77 & 146.14 & 260.75 & 29.67 & - & 923.53 & 224.48 & 26.05 & 1167.05 & 225.79 & - & - & - & 80.56 & - & - \\
\hline Hangzhou & 1.23 & 0.74 & 15.45 & 333.26 & 23.12 & - & 959.79 & 102.66 & 17.97 & 804.96 & 155.74 & - & - & - & 86.57 & - & - \\
\hline Ningbo & 0.71 & 0.80 & 9.56 & 165.95 & - & - & 537.08 & 57.45 & 10.54 & 472.28 & 91.38 & - & - & - & 62.00 & - & - \\
\hline Wenzhou & 2.47 & 0.74 & 6.55 & 112.60 & 14.16 & - & 318.72 & 34.09 & 8.65 & 387.40 & 74.95 & - & - & - & 131.64 & - & - \\
\hline Jiaxing & - & - & 6.65 & 374.83 & - & 58.91 & 429.45 & 45.93 & 22.84 & 1023.31 & 197.99 & - & - & - & - & - & - \\
\hline Huzhou & 0.26 & - & 8.34 & 131.62 & 35.22 & - & 421.54 & 45.09 & 27.78 & 1244.38 & 240.76 & - & - & - & 61.35 & - & - \\
\hline Shaoxing & 0.93 & 0.11 & 12.13 & 193.84 & 11.06 & - & 306.56 & 32.79 & 10.05 & 450.24 & 87.11 & - & - & - & 50.23 & - & - \\
\hline
\end{tabular}


Continued Table S1

\begin{tabular}{|c|c|c|c|c|c|c|c|c|c|c|c|c|c|c|c|c|c|}
\hline & Beef & Cow & Sow & Hog & Goat & Sheep & Layer & Laying duck & Broiler & Meat duck & Goose & Horse & Donkey & Mule & Rabbit & Cattle & Buffalo \\
\hline Jinhua & 2.46 & 1.96 & 11.96 & 265.22 & 8.27 & - & 373.58 & 39.96 & 10.31 & 461.69 & 89.33 & - & - & - & 10.24 & - & - \\
\hline Zhoushan & 0.03 & - & - & 22.32 & - & - & - & - & 0.93 & 41.66 & 8.06 & - & - & - & 6.10 & - & - \\
\hline Taizhou & - & - & 5.80 & 92.15 & 6.40 & - & 244.76 & 26.18 & 10.94 & 489.99 & 94.80 & - & - & - & 58.17 & - & - \\
\hline Lishui & - & - & 4.55 & 86.66 & 7.92 & - & 89.22 & 9.54 & 40.32 & 1806.19 & 349.45 & - & - & - & 11.85 & - & - \\
\hline Quzhou & 1.36 & - & 17.23 & 400.45 & 4.53 & - & 163.31 & 17.47 & 13.13 & 588.25 & 113.81 & - & - & - & - & - & - \\
\hline Hefei & 5.07 & 3.59 & 15.25 & 285.48 & 8.54 & 0.45 & 861.96 & 209.46 & 75.69 & 3390.88 & 656.05 & - & - & - & 3.76 & - & - \\
\hline Huaibei & 3.05 & 0.84 & - & 68.23 & 100.15 & 0.65 & 580.11 & 140.97 & 8.17 & 365.95 & 70.80 & 0.004 & 0.06 & 0.002 & 21.37 & - & - \\
\hline Anqing & 7.09 & - & 17.89 & 284.97 & 7.69 & - & 1631.00 & 444.51 & 29.94 & - & - & - & - & - & 2.00 & - & - \\
\hline Boshou & 13.53 & - & - & 304.66 & 126.99 & - & 438.70 & 106.60 & 11.57 & 518.27 & 100.27 & 0.04 & 0.01 & 0.03 & - & - & - \\
\hline Suzhou & 15.76 & - & - & 483.34 & 241.66 & 3.26 & 1657.91 & 402.88 & 23.10 & 1034.75 & 200.20 & 0.005 & 0.01 & 0.003 & - & - & - \\
\hline Bengbu & 26.67 & - & - & 202.87 & 73.70 & 0.47 & 470.98 & 114.45 & 31.43 & 1408.07 & 272.43 & - & - & - & - & - & - \\
\hline Fuyang & 31.60 & - & - & 560.37 & 138.92 & - & 909.21 & 220.94 & 26.42 & 1183.72 & 229.02 & 0.02 & 0.12 & 0.02 & - & - & - \\
\hline Huainan & 5.05 & - & - & 50.67 & 13.06 & - & 427.94 & 103.99 & 11.30 & 506.30 & 97.96 & - & - & - & - & - & - \\
\hline Chuzhou & 12.72 & - & - & 335.62 & 37.21 & 0.23 & 721.84 & 175.41 & 28.99 & 1298.97 & 251.32 & - & - & - & - & - & - \\
\hline Liu'an & 11.37 & - & - & 405.70 & 46.48 & - & 774.29 & 188.16 & 56.55 & 2533.44 & 490.16 & - & - & - & - & - & - \\
\hline Ma'anshan & 0.20 & - & - & 37.06 & 6.72 & - & 132.64 & 32.23 & 15.78 & 707.14 & 136.81 & - & - & - & - & - & - \\
\hline Wuhu & 2.70 & - & - & 83.15 & 2.92 & - & 516.96 & 125.62 & 25.95 & 1162.41 & 224.90 & - & - & - & - & - & - \\
\hline Xuancheng & 2.30 & - & - & 103.72 & 4.66 & - & 314.63 & 76.46 & 43.47 & 1947.60 & 376.81 & - & - & - & - & - & - \\
\hline Tongling & 0.11 & - & - & 10.24 & - & - & 64.32 & 15.63 & - & - & - & - & - & - & - & - & - \\
\hline Chizhou & 1.07 & - & - & 73.93 & 1.41 & - & 237.85 & 57.80 & 8.54 & 382.44 & 73.99 & - & - & - & - & - & - \\
\hline Huangshan & 0.81 & - & - & 96.38 & 0.49 & 0.03 & 137.06 & 33.31 & 2.14 & 95.73 & 18.52 & 0.03 & 0.003 & 0.004 & - & - & - \\
\hline
\end{tabular}


Table S2. The fertilizer consumption by type and prefectural city in the YRD region, and the provincial-level total consumption from statistics (metric tons).

\begin{tabular}{|c|c|c|c|c|c|c|c|c|c|}
\hline & Urea & $\mathrm{ABC}$ & $\mathrm{AN}$ & AS & DAP & NPK & Other & Total & Statistics \\
\hline Shanghai & 38522.2 & 1819.4 & 44.4 & 6.2 & 2033.7 & 62064.8 & 42.4 & 104533.1 & 89000 \\
\hline Nanjing & 55146.7 & 2871.5 & 91.3 & 12.7 & 2645.4 & 62403.7 & 87.1 & 123258.4 & \\
\hline Wuxi & 52579.2 & 3148.5 & 101.4 & 14.1 & 2347.9 & 65723.9 & 96.8 & 124011.7 & \\
\hline Xuzhou & 172364.1 & 10517.6 & 354.8 & 49.4 & 5962.7 & 158593.2 & 338.6 & 348180.3 & \\
\hline Changzhou & 34264.2 & 2074.6 & 93.4 & 13.0 & 1245.9 & 31052.6 & 89.2 & 68832.9 & \\
\hline Suzhou & 37217.3 & 2230.9 & 57.6 & 8.0 & 1687.8 & 48076.9 & 55.0 & 89333.4 & \\
\hline Nantong & 148372.9 & 7212.5 & 256.6 & 35.8 & 6750.4 & 138864.2 & 245.0 & 301737.4 & \\
\hline Lianyungang & 97247.4 & 5461.8 & 121.6 & 17.0 & 3958.0 & 100200.1 & 116.1 & 207121.9 & \\
\hline Huai'an & 119622.4 & 6238.7 & 170.0 & 23.7 & 4602.1 & 112485.5 & 162.3 & 243304.7 & \\
\hline Yancheng & 222512.3 & 9824.1 & 347.0 & 48.4 & 8286.0 & 236255.7 & 331.2 & 477604.6 & \\
\hline Yangzhou & 74431.4 & 3258.3 & 99.9 & 13.9 & 2118.3 & 73909.4 & 95.4 & 153926.5 & \\
\hline Zhenjiang & 48497.0 & 2494.6 & 83.8 & 11.7 & 1828.2 & 40582.9 & 80.0 & 93578.1 & \\
\hline Taizhou & 114868.5 & 5766.9 & 185.9 & 25.9 & 4691.2 & 106345.4 & 177.5 & 232061.3 & \\
\hline Suqian & 112064.1 & 6159.3 & 162.5 & 22.6 & 3864.9 & 108296.8 & 155.1 & 230725.2 & \\
\hline Jiangsu total & 1289187.3 & 67259.1 & 2125.7 & 296.2 & 49988.8 & 1282790.1 & 2029.1 & 2693676.3 & 2609000 \\
\hline Hangzhou & 33929.4 & 3299.9 & 30.1 & 4.2 & 547.9 & 58816.2 & 28.7 & 96656.4 & \\
\hline Ningbo & 32399.1 & 3521.5 & 35.6 & 5.0 & 831.4 & 56213.0 & 34.0 & 93039.5 & \\
\hline Wenzhou & 21741.3 & 3051.4 & 1.2 & 0.2 & 169.6 & 33691.6 & 1.1 & 58656.3 & \\
\hline Jiaxing & 37957.0 & 4132.5 & 35.5 & 5.0 & 803.5 & 57789.3 & 33.9 & 100756.7 & \\
\hline Huzhou & 28860.3 & 3742.5 & 26.0 & 3.6 & 576.3 & 35435.9 & 24.8 & 68669.4 & \\
\hline Shaoxing & 33122.2 & 3801.7 & 35.2 & 4.9 & 768.2 & 46923.1 & 33.6 & 84688.9 & \\
\hline Jinhua & 28634.6 & 3062.7 & 30.3 & 4.2 & 689.9 & 39802.2 & 28.9 & 72252.8 & \\
\hline Zhoushan & 2736.0 & 238.6 & 4.1 & 0.6 & 84.7 & 5136.9 & 4.0 & 8204.9 & \\
\hline Taizhou & 27127.9 & 2912.9 & 31.8 & 4.4 & 500.1 & 46903.3 & 30.4 & 77510.8 & \\
\hline Lishui & 20953.3 & 2403.5 & 36.6 & 5.1 & 563.7 & 32309.7 & 34.9 & 56306.8 & \\
\hline Quzhou & 27943.3 & 3597.7 & 21.1 & 2.9 & 461.3 & 33127.1 & 20.2 & 65173.5 & \\
\hline Zhejiang total & 295404.4 & 33764.7 & 287.6 & 40.1 & 5996.5 & 446148.3 & 274.5 & 781916.0 & 717000 \\
\hline Hefei & 89980.7 & 3144.9 & 32.4 & 4.5 & 4567.2 & 115602.5 & 31.0 & 213363.1 & \\
\hline Huaibei & 26884.4 & 678.8 & 12.2 & 1.7 & 897.6 & 42907.1 & 11.7 & 71393.5 & \\
\hline
\end{tabular}


Continued Table S2

\begin{tabular}{cccccccccc}
\hline Anqing & 104403.0 & 2946.8 & 34.1 & 4.7 & 5869.2 & 120970.4 & 32.5 & 234260.7 \\
Bozhou & 112979.8 & 2989.4 & 68.5 & 9.6 & 5221.0 & 182035.0 & 65.4 & 303368.7 & \\
Suzhou & 104919.7 & 2576.0 & 35.6 & 5.0 & 3810.2 & 151179.4 & 34.0 & 262559.9 & \\
Bengbu & 72865.7 & 1953.0 & 36.4 & 5.1 & 4875.7 & 119840.0 & 34.8 & 199610.6 & \\
Fuyang & 139596.0 & 3727.8 & 75.8 & 10.6 & 5704.0 & 223240.0 & 72.4 & 372426.6 & \\
Huainan & 29430.1 & 845.1 & 14.8 & 2.1 & 1092.6 & 48097.9 & 14.2 & 79496.8 & \\
Chuzhou & 115575.2 & 2824.0 & 53.9 & 7.5 & 5953.7 & 176377.9 & 51.5 & 300843.7 & \\
Liuan & 112162.7 & 2719.9 & 36.0 & 5.0 & 3482.5 & 155355.5 & 34.4 & 273796.0 & \\
Maanshan & 28821.6 & 721.0 & 10.6 & 1.5 & 1237.6 & 39867.0 & 10.1 & 70669.3 & \\
Wuhu & 46288.4 & 1159.8 & 17.4 & 2.4 & 2845.1 & 61312.2 & 16.6 & 111641.8 & \\
Xuancheng & 41839.9 & 1015.9 & 15.7 & 2.2 & 1652.2 & 56933.7 & 15.0 & 101474.6 & \\
Tongling & 5782.1 & 141.1 & 1.9 & 0.3 & 319.6 & 7754.7 & 1.8 & 14001.5 & \\
Chizhou & 26140.2 & 559.0 & 4.6 & 0.6 & 1588.7 & 29744.6 & 4.4 & 58042.0 & \\
Huangshan & 15024.7 & 364.5 & 8.3 & 1.2 & 594.2 & 19994.1 & 7.9 & 35994.8 & \\
Anhui total & $\mathbf{1 0 7 2 6 9 4 . 0}$ & $\mathbf{2 8 3 6 6 . 9}$ & $\mathbf{4 5 8 . 3}$ & $\mathbf{6 3 . 9}$ & $\mathbf{4 9 7 1 1 . 1}$ & $\mathbf{1 5 5 1 2 1 1 . 9}$ & $\mathbf{4 3 7 . 5}$ & $\mathbf{2 7 0 2 9 4 3 . 6}$ & $\mathbf{2 7 3 1 0 2 5}$ \\
& & & & & & & & & \\
YRD total & $\mathbf{2 6 9 5 8 0 7 . 8}$ & $\mathbf{1 3 1 2 1 0 . 1}$ & $\mathbf{2 9 1 6 . 1}$ & $\mathbf{4 0 6 . 4}$ & $\mathbf{1 0 7 7 3 0 . 2}$ & $\mathbf{3 3 4 2 2 1 5 . 0}$ & $\mathbf{2 7 8 3 . 4}$ & $\mathbf{6 2 8 3 0 6 9 . 0}$ & $\mathbf{6 1 4 6 0 2 5}$ \\
\hline
\end{tabular}

Note: ABC, AN, AS, DAP, and NPK represent ammonium bicarbonate, ammonium nitrate, ammonium sulfate, diammonium phosphate and complex-fertilizer, respectively. 
Table S3. The $\mathrm{NH}_{3}$ emission factors used in E1.

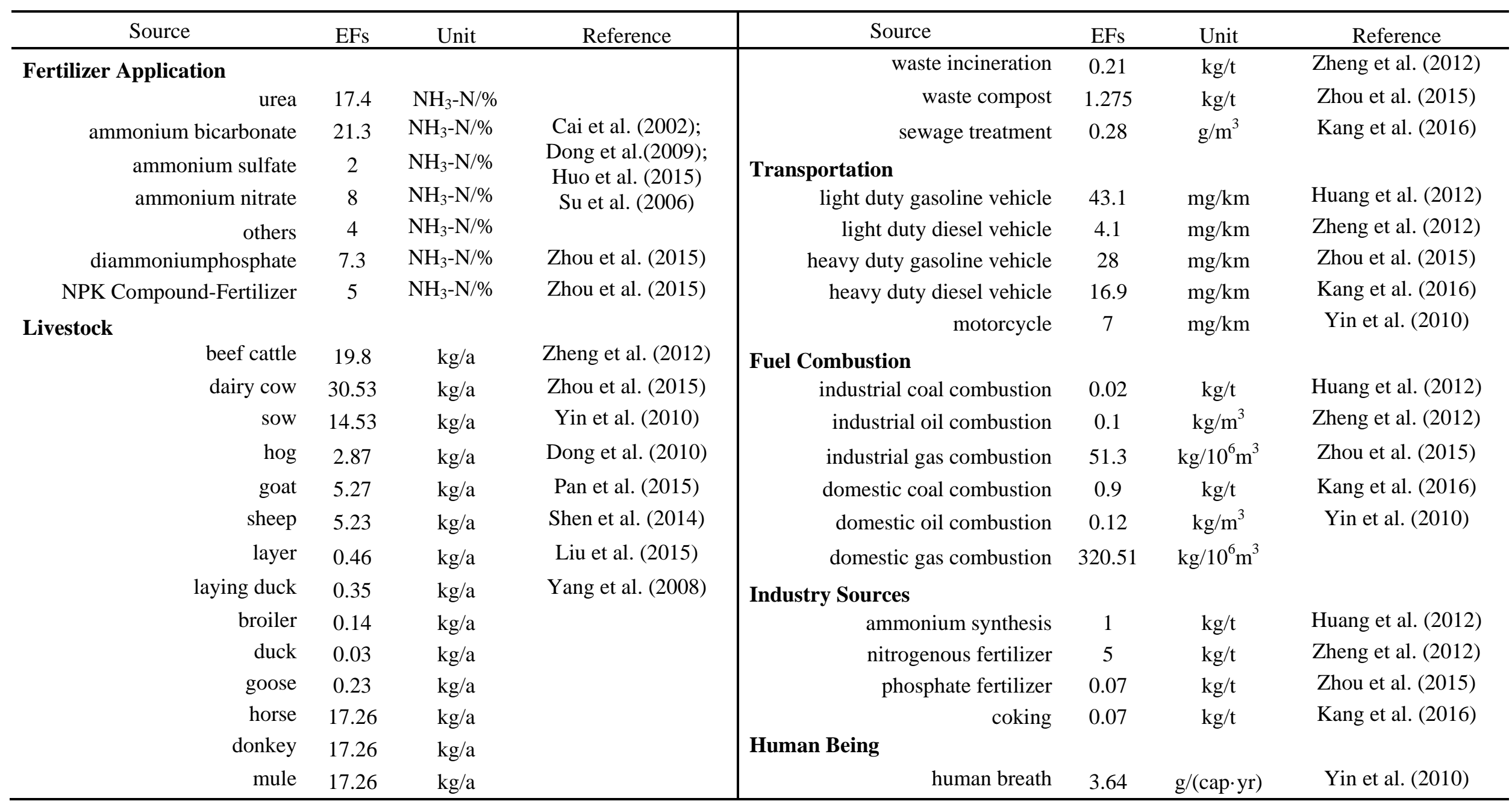


Table S4. The relevant parameters used for correction of emission factors of fertilizer activity in E2.

\begin{tabular}{ccccccccc}
\hline \multirow{2}{*}{ Fertilizer Categories } & \multicolumn{2}{c}{$\mathrm{pH}$} & & \multicolumn{2}{c}{$\mathrm{T}$} & & \multirow{2}{*}{$\mathrm{CF}_{\text {rate }}$} & $\mathrm{CF}_{\text {method }}$ \\
\cline { 2 - 3 } & $\mathrm{pH}$ slope & $\mathrm{pH}$ intercept & & $\mathrm{T}_{\text {basal }}$ & $\mathrm{T}$ slope & & \\
\hline Urea & 6.265 & -25.029 & & 27.6 & 0.35 & 1.18 & 0.32 \\
$\mathrm{ABC}$ & 6.147 & -14.994 & & 27.6 & 0.44 & 1.18 & 0.32 \\
$\mathrm{AN}$ & 1.793 & -10.45 & & 13 & 0.06 & 1.18 & 0.32 \\
AS & 0 & 0.8 & & 0 & 0.01 & 1.18 & 0.32 \\
Other nitrogen fertilizers & 0 & 0.8 & & 0 & 0.01 & 1.18 & 0.32 \\
DAP & 0 & 0.8 & & 0 & 0.01 & 1.18 & 0.32 \\
NPK & 2.806 & -10.158 & & 13 & 0.01 & 1.18 & 0.32 \\
\hline
\end{tabular}


Table S5. The arameters used in estimates of annual TAN excretion per cattle.

\begin{tabular}{|c|c|c|c|c|c|c|c|c|c|}
\hline & \multirow{2}{*}{$\begin{array}{c}\text { Raising } \\
\text { cycle }\end{array}$} & \multicolumn{2}{|c|}{ Excretion (kg/cattle/day) } & \multicolumn{2}{|c|}{ Nitrogen content (\%) } & \multirow{2}{*}{$\begin{array}{c}\text { TAN } \\
(\%)\end{array}$} & \multicolumn{3}{|c|}{ Annual TAN (tons/cattle) } \\
\hline & & Urine & Excrement & Urine & Excrement & & Urine & Excrement & Total \\
\hline Beef & 365 & 7.5 & 13.5 & 0.9 & 0.38 & 60 & 0.0148 & 0.0112 & 0.0260 \\
\hline Cow & 365 & 12 & 23.5 & 0.9 & 0.38 & 60 & 0.0237 & 0.0196 & 0.0432 \\
\hline Horse & 365 & 6.5 & 15 & 1.4 & 0.2 & 60 & 0.0199 & 0.0066 & 0.0265 \\
\hline Donkey & 365 & 6.5 & 15 & 1.4 & 0.2 & 60 & 0.0199 & 0.0066 & 0.0265 \\
\hline Mule & 365 & 6.5 & 15 & 1.4 & 0.2 & 60 & 0.0199 & 0.0066 & 0.0265 \\
\hline Sow & 365 & 5.7 & 2.1 & 0.4 & 0.34 & 70 & 0.0058 & 0.0018 & 0.0076 \\
\hline Hog & 75 & 3.2 & 1.5 & 0.4 & 0.34 & 70 & 0.0007 & 0.0003 & 0.0009 \\
\hline Goat & 365 & 0.705 & 2.05 & 1.35 & 0.75 & 55 & 0.0019 & 0.0031 & 0.0050 \\
\hline Sheep & 365 & 0.705 & 2.05 & 1.35 & 0.75 & 55 & 0.0019 & 0.0031 & 0.0050 \\
\hline Layer & 365 & - & 0.12 & - & 1.63 & 70 & - & 0.0005 & 0.0005 \\
\hline Laying duck & 365 & - & 0.13 & - & 1.1 & 70 & - & 0.0004 & 0.0004 \\
\hline Broiler & 50 & - & 0.09 & - & 1.63 & 70 & - & $5 \times 10^{-5}$ & $5 \times 10^{-5}$ \\
\hline Meat duck & 55 & - & 0.1 & - & 1.1 & 70 & - & $4 \times 10^{-5}$ & $4 \times 10^{-5}$ \\
\hline Goose & 70 & - & 0.1 & - & 0.55 & 70 & - & $3 \times 10^{-5}$ & $3 \times 10^{-5}$ \\
\hline Rabbit & 55 & 0.3 & 0.15 & 0.15 & 1.72 & 45 & - & $6 \times 10^{-5}$ & $7 \times 10^{-5}$ \\
\hline Cattle & 365 & 10 & 18 & 0.9 & 0.6 & 50 & 0.0164 & 0.01971 & 0.0361 \\
\hline Buffalo & 365 & 10 & 18 & 0.9 & 0.6 & 45 & 0.0148 & 0.0177 & 0.0325 \\
\hline
\end{tabular}


Table S6. The temperature-dependant emission factors by stage/phase in livestock farming in E2 (\% TAN).

\begin{tabular}{|c|c|c|c|c|c|c|c|c|c|c|c|c|c|c|c|c|c|}
\hline & \multirow[b]{2}{*}{$\mathrm{EF}_{\text {outdoors }}$} & \multicolumn{3}{|c|}{ EF $_{\text {Housing }}$ (liquid) } & \multicolumn{3}{|c|}{$\mathrm{EF}_{\text {Housing }}$ (solid) } & \multicolumn{4}{|c|}{$\mathrm{EF}_{\text {Storage }}$ (liquid) } & \multicolumn{4}{|c|}{$\mathrm{EF}_{\text {Storage }}$ (solid) } & \multirow{2}{*}{$\begin{array}{l}\mathrm{EF}_{\text {spreading }} \\
\text { (liquid) }\end{array}$} & \multirow{2}{*}{$\begin{array}{c}\mathrm{EF}_{\text {spreading }} \\
\text { (solid) }\end{array}$} \\
\hline & & $<10^{\circ} \mathrm{C}$ & $10-20{ }^{\circ} \mathrm{C}$ & $>20{ }^{\circ} \mathrm{C}$ & $<10^{\circ} \mathrm{C}$ & $10-20{ }^{\circ} \mathrm{C}$ & $>20{ }^{\circ} \mathrm{C}$ & $\mathrm{NH}_{3}$ & $\mathrm{~N}_{2} \mathrm{O}$ & NO & $\mathrm{N}_{2}$ & $\mathrm{NH}_{3}$ & $\mathrm{~N}_{2} \mathrm{O}$ & NO & $\mathrm{N}_{2}$ & & \\
\hline \multicolumn{18}{|c|}{ Free-range } \\
\hline Beef & 53 & 7 & 10.5 & 14 & 7 & 10.5 & 14 & 20 & 1 & 0 & 0.3 & 27 & 8 & 1 & 30 & 55 & 79 \\
\hline Cow & 41.5 & 7 & 10.5 & 14 & 7 & 10.5 & 14 & 20 & 1 & 0 & 0.3 & 27 & 8 & 1 & 30 & 55 & 79 \\
\hline Horse & 0 & 9.3 & 14 & 18.7 & 9.3 & 14 & 18.7 & 35 & 0 & 0 & 0.3 & 35 & 8 & 1 & 30 & 90 & 81 \\
\hline Donkey & 0 & 9.3 & 14 & 18.7 & 9.3 & 14 & 18.7 & 35 & 0 & 0 & 0.3 & 35 & 8 & 1 & 30 & 90 & 81 \\
\hline Mule & 0 & 9.3 & 14 & 18.7 & 9.3 & 14 & 18.7 & 35 & 0 & 0 & 0.3 & 35 & 8 & 1 & 30 & 90 & 81 \\
\hline Sow & 0 & 9.2 & 14.7 & 20.2 & 9.2 & 14.7 & 20.2 & 14 & 0 & 0 & 0.3 & 45 & 5 & 1 & 30 & 40 & 81 \\
\hline Hog & 0 & 6.2 & 10.2 & 14.2 & 6.2 & 10.2 & 14.2 & 14 & 0 & 0 & 0.3 & 45 & 5 & 1 & 30 & 40 & 81 \\
\hline Goat & 64 & 7 & 10.5 & 14 & 7 & 10.5 & 14 & 24 & 4 & 0 & 0.3 & 27.5 & 7.5 & 1 & 30 & 72.5 & 80 \\
\hline Sheep & 64 & 7 & 10.5 & 14 & 7 & 10.5 & 14 & 24 & 4 & 0 & 0.3 & 27.5 & 7.5 & 1 & 30 & 72.5 & 80 \\
\hline Layer & 69 & 24.9 & 45.2 & 56.5 & 24.9 & 45.2 & 56.5 & 0 & 0 & 0 & 0 & 14 & 4 & 1 & 30 & 0 & 63 \\
\hline Laying duck & 54 & 24.9 & 45.2 & 56.5 & 24.9 & 45.2 & 56.5 & 0 & 0 & 0 & 0 & 24 & 3 & 1 & 30 & 0 & 63 \\
\hline Broiler & 66 & 22.2 & 40.3 & 50.4 & 22.2 & 40.3 & 50.4 & 0 & 0 & 0 & 0 & 17 & 3 & 1 & 30 & 0 & 63 \\
\hline Meat duck & 54 & 22.2 & 40.3 & 50.4 & 22.2 & 40.3 & 50.4 & 0 & 0 & 0 & 0 & 24 & 3 & 1 & 30 & 0 & 63 \\
\hline Goose & 54 & 22.2 & 40.3 & 50.4 & 22.2 & 40.3 & 50.4 & 0 & 0 & 0 & 0 & 24 & 3 & 1 & 30 & 0 & 63 \\
\hline \multicolumn{18}{|c|}{ Intensive } \\
\hline Beef & 53 & 7 & 10.5 & 14 & 7 & 10.5 & 14 & 16 & 1 & 0 & 0.3 & 4.2 & 8 & 1 & 30 & 55 & 79 \\
\hline Cow & 41.5 & 7 & 10.5 & 14 & 7 & 10.5 & 14 & 16 & 1 & 0 & 0.3 & 4.2 & 8 & 1 & 30 & 55 & 79 \\
\hline Horse & 0 & 9.3 & 14 & 18.7 & 9.3 & 14 & 18.7 & 16 & 0 & 0 & 0.3 & 4.2 & 8 & 1 & 30 & 90 & 81 \\
\hline Donkey & 0 & 9.3 & 14 & 18.7 & 9.3 & 14 & 18.7 & 16 & 0 & 0 & 0.3 & 4.2 & 8 & 1 & 30 & 90 & 81 \\
\hline Mule & 0 & 9.3 & 14 & 18.7 & 9.3 & 14 & 18.7 & 16 & 0 & 0 & 0.3 & 4.2 & 8 & 1 & 30 & 90 & 81 \\
\hline
\end{tabular}


Continued Table S6

\begin{tabular}{|c|c|c|c|c|c|c|c|c|c|c|c|c|c|c|c|c|c|}
\hline & \multirow[b]{2}{*}{$\mathrm{EF}_{\text {outdoors }}$} & \multicolumn{3}{|c|}{$\mathrm{EF}_{\text {Housing }}$ (liquid) } & \multicolumn{3}{|c|}{$\mathrm{EF}_{\text {Housing }}$ (solid) } & \multicolumn{4}{|c|}{$\mathrm{EF}_{\text {Storage }}$ (liquid) } & \multicolumn{4}{|c|}{$\mathrm{EF}_{\text {Storage }}$ (solid) } & \multirow{2}{*}{$\begin{array}{c}\mathrm{EF}_{\text {spreading }} \\
\text { (liquid) }\end{array}$} & \multirow{2}{*}{$\begin{array}{c}\mathrm{EF}_{\text {spreading }} \\
\text { (solid) }\end{array}$} \\
\hline & & $<10^{\circ} \mathrm{C}$ & $10-20{ }^{\circ} \mathrm{C}$ & $>20^{\circ} \mathrm{C}$ & $<10^{\circ} \mathrm{C}$ & $10-20^{\circ} \mathrm{C}$ & $>20^{\circ} \mathrm{C}$ & $\mathrm{NH}_{3}$ & $\mathrm{~N}_{2} \mathrm{O}$ & NO & $\mathrm{N}_{2}$ & $\mathrm{NH}_{3}$ & $\mathrm{~N}_{2} \mathrm{O}$ & NO & $\mathrm{N}_{2}$ & & \\
\hline \multicolumn{18}{|c|}{ Intensive } \\
\hline Sow & 0 & 8.9 & 14.3 & 19.7 & 8.9 & 14.3 & 19.7 & 3.8 & 0 & 0 & 0.3 & 4.6 & 5 & 1 & 30 & 40 & 81 \\
\hline Hog & 0 & 11.3 & 18.5 & 25.7 & 11.3 & 18.5 & 25.7 & 3.8 & 0 & 0 & 0.3 & 4.6 & 5 & 1 & 30 & 40 & 81 \\
\hline Goat & 64 & 7 & 10.5 & 14 & 7 & 10.5 & 14 & 16 & 4 & 0 & 0.3 & 4.2 & 7.5 & 1 & 30 & 72.5 & 80 \\
\hline Sheep & 64 & 7 & 10.5 & 14 & 7 & 10.5 & 14 & 16 & 4 & 0 & 0.3 & 4.2 & 7.5 & 1 & 30 & 72.5 & 80 \\
\hline Layer & 69 & 0 & 0 & 0 & 19.7 & 35.9 & 44.9 & 0 & 0 & 0 & 0 & 3.7 & 4 & 1 & 30 & 0 & 63 \\
\hline Laying duck & 54 & 0 & 0 & 0 & 19.7 & 35.9 & 44.9 & 0 & 0 & 0 & 0 & 3.7 & 3 & 1 & 30 & 0 & 63 \\
\hline Broiler & 66 & 0 & 0 & 0 & 22.2 & 40.3 & 50.4 & 0 & 0 & 0 & 0 & 0.8 & 3 & 1 & 30 & 0 & 63 \\
\hline Meat duck & 54 & 0 & 0 & 0 & 22.2 & 40.3 & 50.4 & 0 & 0 & 0 & 0 & 0.8 & 3 & 1 & 30 & 0 & 63 \\
\hline Goose & 54 & 0 & 0 & 0 & 22.2 & 40.3 & 50.4 & 0 & 0 & 0 & 0 & 0.8 & 3 & 1 & 30 & 0 & 63 \\
\hline
\end{tabular}


Table S7. Model performance for meteorological parameters in D2.

\begin{tabular}{|c|c|c|c|c|c|c|}
\hline Parameters & Indicator & January & April & July & October & Benchmark \\
\hline \multirow[t]{8}{*}{ Wind speed } & Mean OBS $(\mathrm{m} / \mathrm{s})$ & 2.50 & 2.62 & 2.52 & 2.64 & \\
\hline & Mean SIM (m/s) & 2.55 & 2.52 & 2.38 & 2.63 & \\
\hline & Bias (m/s) & 0.05 & -0.10 & -0.14 & -0.009 & $\leq \pm 0.5$ \\
\hline & RMSE (m/s) & 0.27 & 0.29 & 0.33 & 0.27 & $\leq 2.0$ \\
\hline & IOA & 0.95 & 0.94 & 0.94 & 0.97 & $\geq 0.6$ \\
\hline & $\mathrm{R}^{2}$ & 0.82 & 0.82 & 0.82 & 0.89 & \\
\hline & NMB (\%) & 1.91 & -3.94 & -5.53 & -0.32 & \\
\hline & NME (\%) & 8.37 & 9.10 & 10.53 & 7.75 & \\
\hline \multirow[t]{8}{*}{ Wind direction } & Mean OBS $\left(^{\circ}\right)$ & 184.29 & 163.29 & 169.28 & 174.34 & \\
\hline & Mean SIM $\left(^{\circ}\right)$ & 165.37 & 147.09 & 159.80 & 155.66 & \\
\hline & Bias $\quad\left(^{\circ}\right)$ & -18.91 & -15.68 & -9.17 & -18.68 & $\leq \pm 10$ \\
\hline & $\operatorname{RMSE}\left({ }^{\circ}\right)$ & 33.88 & 27.82 & 23.15 & 30.93 & \\
\hline & IOA & 0.87 & 0.88 & 0.87 & 0.92 & \\
\hline & $\mathrm{R}^{2}$ & 0.69 & 0.74 & 0.63 & 0.81 & \\
\hline & NMB (\%) & -10.26 & -9.92 & -5.60 & -10.72 & \\
\hline & NME (\%) & 13.95 & 13.41 & 9.62 & 13.91 & \\
\hline \multirow[t]{8}{*}{ Temperature } & Mean OBS $\left({ }^{\circ} \mathrm{C}\right)$ & 5.60 & 16.30 & 27.33 & 18.69 & \\
\hline & Mean SIM $\left({ }^{\circ} \mathrm{C}\right)$ & 7.09 & 16.03 & 26.64 & 18.75 & \\
\hline & $\operatorname{Bias}\left({ }^{\circ} \mathrm{C}\right)$ & 1.49 & -0.26 & -0.66 & 0.06 & $\leq 0.5$ \\
\hline & $\operatorname{RMSE}\left({ }^{\circ} \mathrm{C}\right)$ & 1.76 & 0.95 & 1.27 & 0.69 & \\
\hline & IOA & 0.95 & 0.98 & 0.95 & 0.99 & $\geq 0.7$ \\
\hline & $\mathrm{R}^{2}$ & 0.95 & 0.94 & 0.89 & 0.96 & \\
\hline & NMB (\%) & 26.60 & -1.62 & -2.51 & 0.34 & \\
\hline & NME (\%) & 28.00 & 4.70 & 3.57 & 2.94 & \\
\hline \multirow[t]{8}{*}{ Relative humidity } & Mean OBS (\%) & 65.16 & 75.69 & 79.81 & 71.96 & \\
\hline & Mean SIM (\%) & 61.87 & 76.21 & 82.24 & 70.80 & \\
\hline & Bias (\%) & -3.29 & 0.51 & 2.36 & -1.16 & \\
\hline & RMSE (\%) & 6.61 & 3.86 & 4.24 & 4.07 & \\
\hline & IOA & 0.97 & 0.98 & 0.95 & 0.98 & $\geq 0.7$ \\
\hline & $\mathrm{R}^{2}$ & 0.92 & 0.95 & 0.89 & 0.94 & \\
\hline & NMB (\%) & -5.06 & 0.69 & 3.05 & -1.61 & \\
\hline & NME (\%) & 8.42 & 4.23 & 4.29 & 4.15 & \\
\hline
\end{tabular}

Note: OBS and SIM indicate the results from observation and simulation, respectively. The Bias, IOA, RMSE, NMB and NME were calculated using following equations ( $\mathrm{P}$ and $\mathrm{O}$ indicates the results from modeling prediction and observation, respectively):

$$
\begin{aligned}
& \text { Bias }=\frac{1}{n} \sum_{i=1}^{n}\left(P_{i}-O_{i}\right) ; I O A=1-\frac{\sum_{i=1}^{n}\left(P_{i}-O_{i}\right)^{2}}{\sum_{i=1}^{n}\left(\left|P_{i}-\bar{O}\right|+\left|O_{i}-\bar{O}\right|\right)^{2}} ; R M S E=\sqrt{\frac{1}{n} \sum_{i=1}^{n}\left(P_{i}-O_{i}\right)^{2}} \\
& N M B=\frac{\sum_{i=1}^{n}\left(P_{i}-O_{i}\right)}{\sum_{i=1}^{n} O_{i}} \times 100 \% ; \quad N M E=\frac{\sum_{i=1}^{n}\left|P_{i}-O_{i}\right|}{\sum_{i=1}^{n} O_{i}} \times 100 \%
\end{aligned}
$$


Table S8. The inter-annual change in $\mathrm{SO}_{2}$ and $\mathrm{NO}_{2}$ VCDs for the YRD region 2012-2014 (\%)

\begin{tabular}{cccccc}
\hline & Jiangsu & Zhejiang & Anhui & Shanghai & Total YRD \\
\hline $\mathrm{SO}_{2}$ & -49.94 & -29.53 & -51.38 & -56.72 & -47.84 \\
$\mathrm{NO}_{2}$ & -29.12 & -26.22 & -35.69 & -29.48 & -30.91 \\
\hline
\end{tabular}


Figure S1.The $\mathrm{NH}_{3}$ volatilization of urea and ABC application in E2
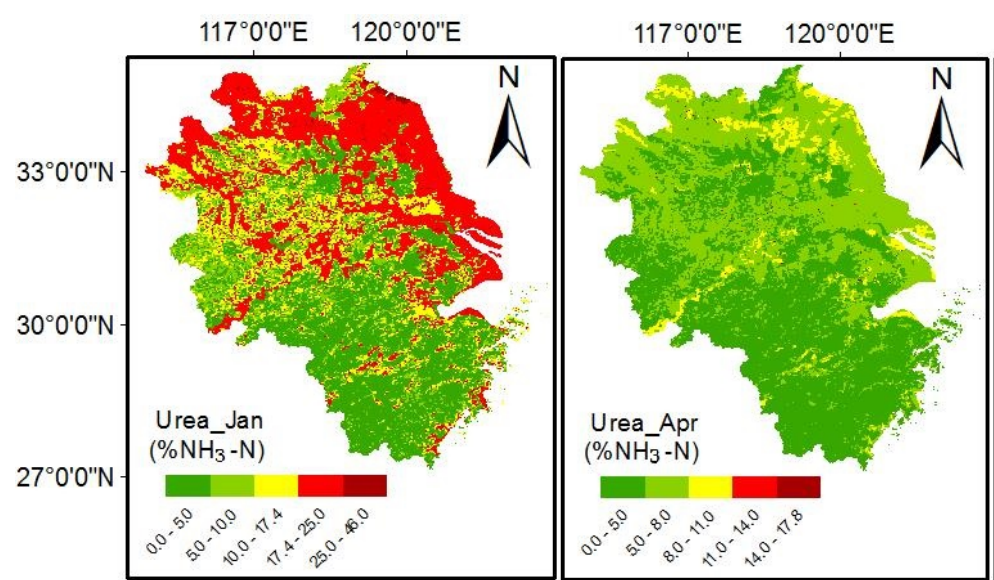

$117^{\circ} 0^{\prime} 0^{\prime \prime E} \quad 120^{\circ} 0^{\prime} 0^{\prime \prime} \mathrm{E}$

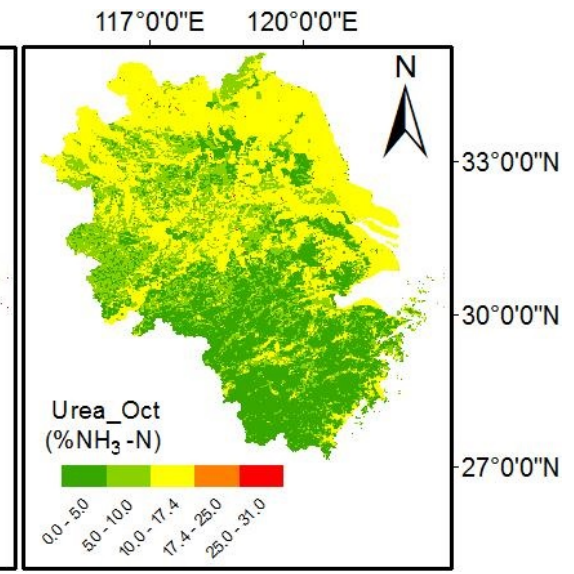

(a) $\mathrm{NH}_{3}$ volatility of urea

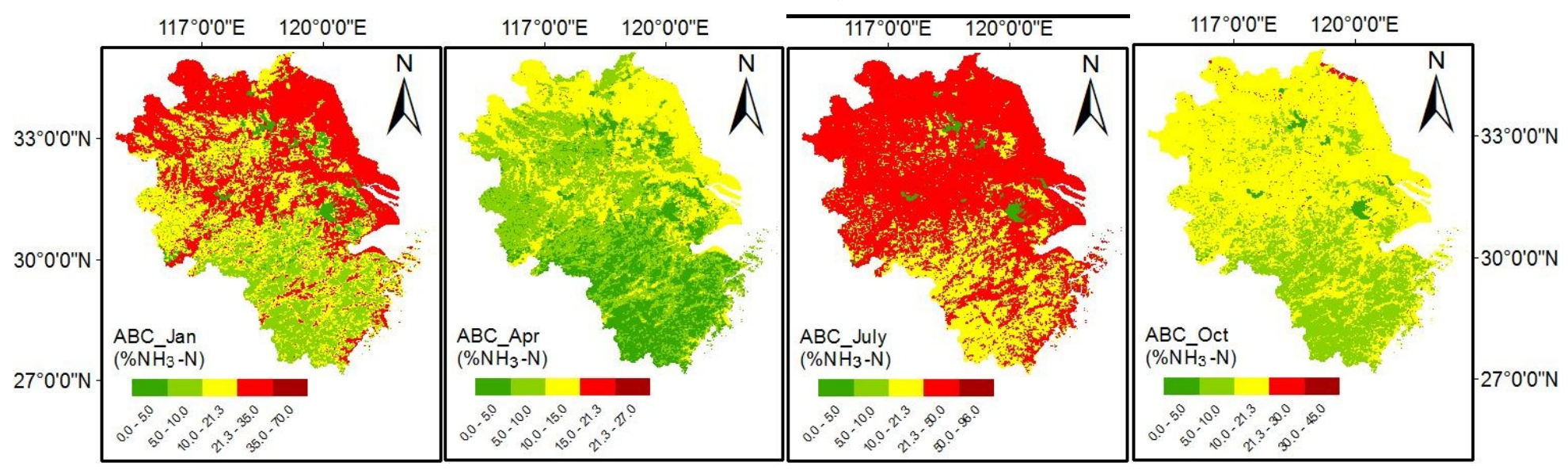

(b) $\mathrm{NH}_{3}$ volatility of $\mathrm{ABC}$ 
Figure S2. Total ammoniacal nitrogen (TAN) for three main raising systems (taken from Huang et al., 2012)

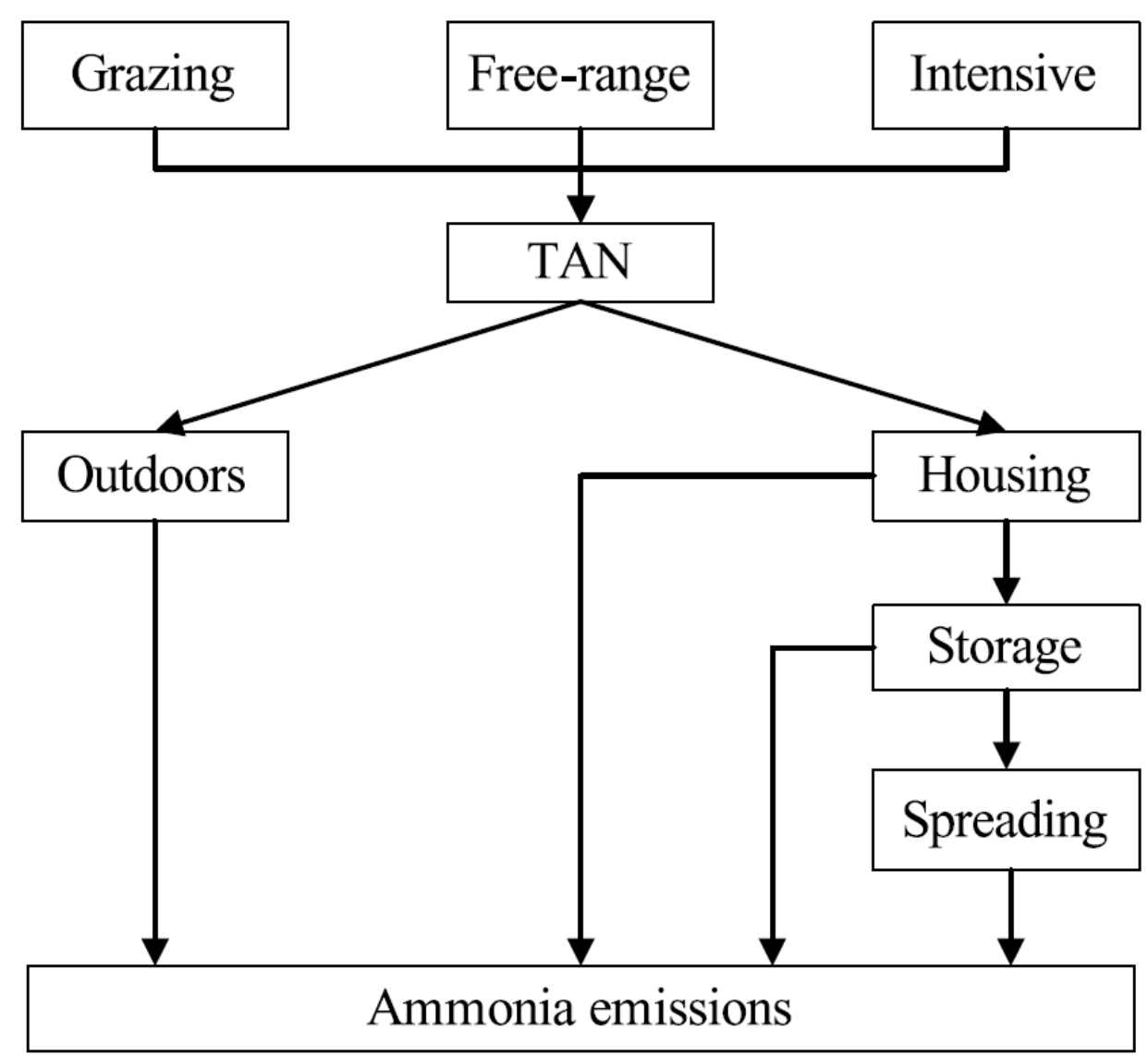


Figure S3. The daily $\mathrm{NH}_{3}$ concentration at JSPAES for October 2014 from observation and simulation with E1 and E2.

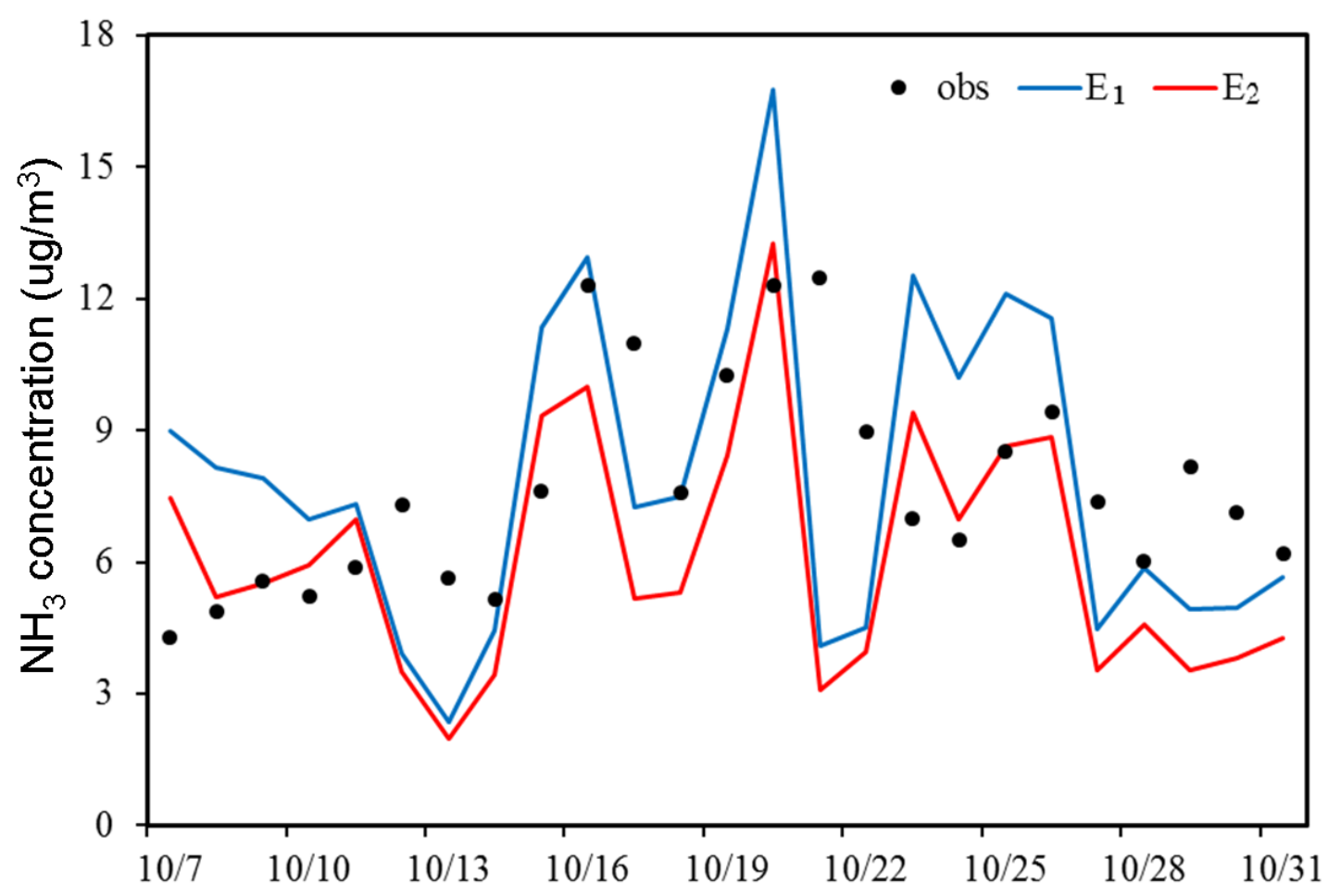


Figure S4. The $\mathrm{NH}_{3}$ volatilization rates under different soil $\mathrm{pH}$ values for urea (a) and ABC fertilizer (b). The blue dots indicate the values by grid in the YRD region in E2, while the red dots indicate the results from the field measurements by Zhong et al. (2006) (a) and Zhang et al. (2002) (b). The solid lines and equations were obtained from linear regression for the gridded values in the YRD region in E2.

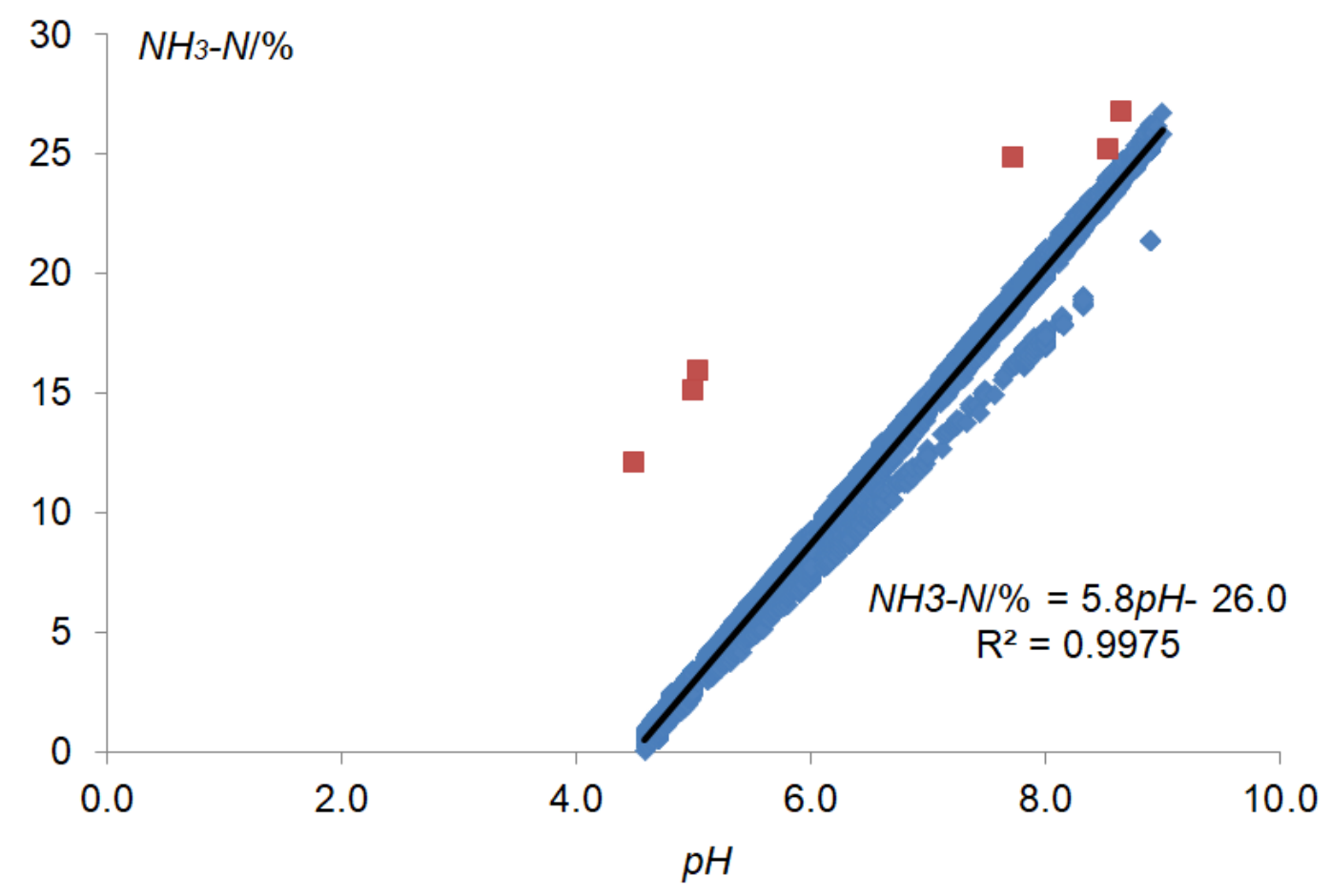

(a) Urea

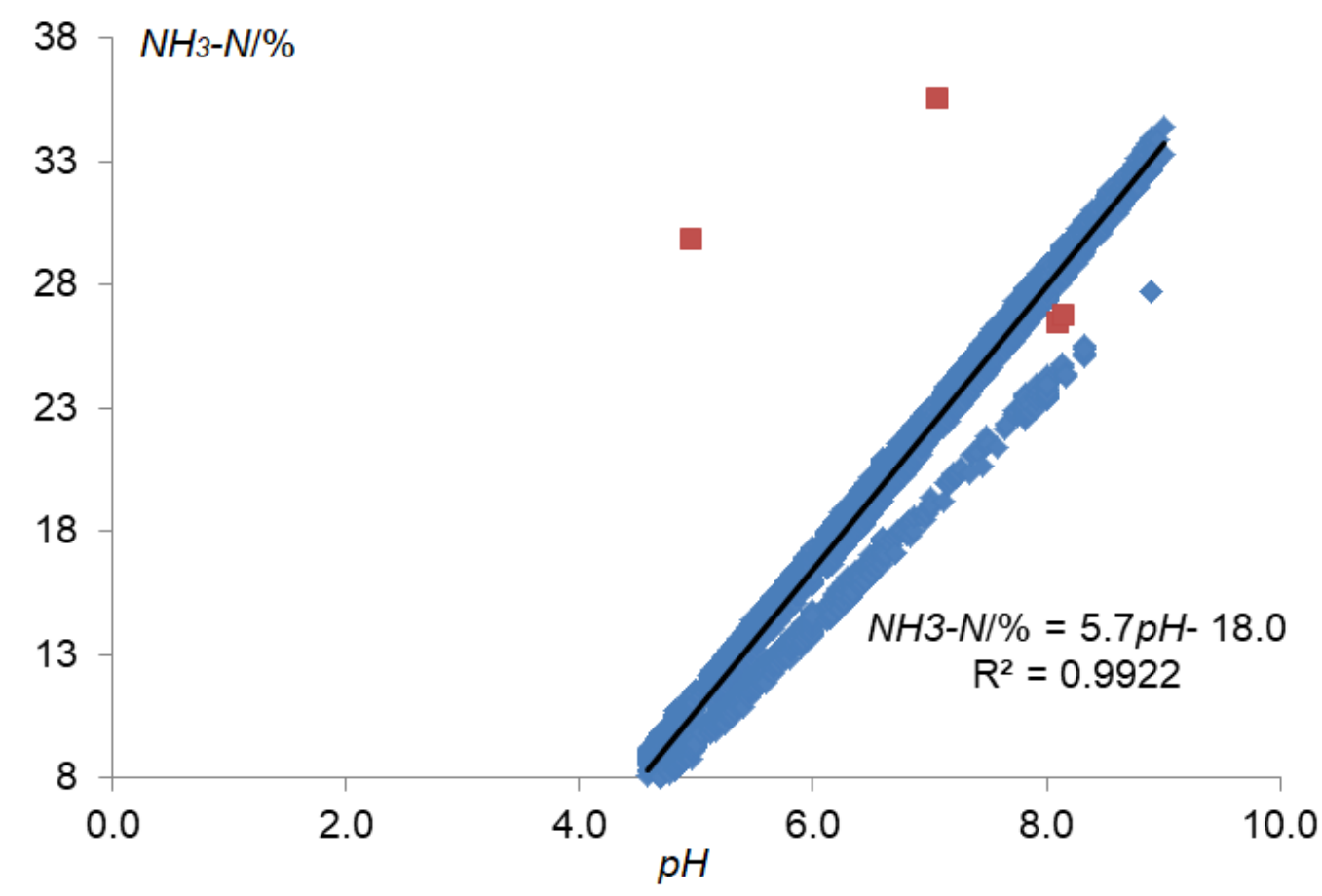

(b) $\mathrm{ABC}$ 


\section{References}

Cai, G. X., Chen, D. L., Ding, H., Pacholski, A., Fan, X. H., Zhu, Z. L.: Nitrogen losses from fertilizers applied to maize, wheat and rice in the North China Plain. Nutr. Cycl. Agroecosys., 63, 187-195, 2002.

Dong, W., Xin, J., Wang, S.: Temporal and spatial distribution of anthropogenic ammonia emissions in China: 1994-2006, Environ. Sci., 31, 1457-1463, 2010 (in Chinese).

Dong, Y., Chen, C., Huang, C., Wang, H., Li, L., Dai, P., Jia, J.: Anthropogenic emissions and distribution of ammonia over the Yangtze River Delta, Acta Scientiae Circumstantiae, 29, 1611-1617, 2009.

Huang, X., Song, Y., Li, M., Li, J., Huo, Q., Cai, X., Zhu, T., Hu, M., Zhang, H.: A high-resolution ammonia emission inventory in China. Global Biogeochem. Cy., 26, GB1030, doi: 10.1029/2011GB004161, 2012.

Huo, Q., Cai, X., Kang, L., Zhang, H., Song, Y., Zhu, T.: Estimating ammonia emissions from a winter wheat cropland in North China Plain with field experiments and inverse dispersion modeling, Atmos. Environ., 104, 1-10, 2015.

Liu, C., Yao, L.: Agricultural ammonia emission inventory and its distribution characteristics in Jiangsu Province, Journal of Anhui Agri. Sci., 44, 70-74, 2016 (in Chinese).

Kang, Y., Liu, M., Song, Y., Huang, X., Yao, H., Cai, X., Zhang, H., Kang, L., Liu, X., Yan, X., He, H., Zhang, Q., Shao, M., Zhu, T.: High-resolution ammonia emissions inventories in China from 1980 to 2012, Atmos. Chem. Phys., 16, 2043-2058, 2016.

Pan, T., Xue, N., Sun, C., Liu, G., Zhang, J., Li, S.: Distribution characteristics of ammonia emission from livestock farming industry in Beijing, Environ. Sci. Technol., 38, 159-162, 2015.

Shen, X., Yin, S., Zheng, J., Lu, Q., Zhong, L.: Anthropogenic ammonia emission inventory and its mitigation potential in Guangdong Province, Acta Scientiae Circumstantiae, 34, 43-53, 2014.

Su, F., Huang, B., Ding, X., Gao, Z., Chen, X., Zhang, F., Kogge, M., Römheld, V.: Ammonia volatilization of different nitrogen fertilizer types, Soils, 38, 682-686, 2006 (in Chinese).

Yang, Z.: Estimation of ammonia emission from livestock in China based on mass-flow method and regional comparison, Master thesis, Peking University, Beijing, China, 2008.

Yin, S., Zheng, J., Zhang, L., Zhong, L.: Anthropogenic ammonia emission inventory and characteristics in the Pearl River Delta region, Environ. Sci., 31, 1146-1151, 2010.

Zhang, Q., Zhang, M., Yang, Y., Lu, J.: Volatilization of ammonium bicarbonate and urea in main soil of Shandong Province, Chinese Journal of Soil Science, 33, 32-34, 2002.

Zheng, J., Yin, S., Kang, D., Che, W., Zhong, L.: Development and uncertainty analysis of a high-resolution $\mathrm{NH}_{3}$ emission inventory and its implications with precipitation over the Pearl River Delta region, China, Atmos. Chem. Phys., 12, 7041-7058, 2012. 
Zhong, N., Zeng, Q., Zhang, L., Liao, B., Zhou, X., Jiang, J.: Effects of acidity and alkalinity on urea transformation in soil, Chinese Journal of Soil Science, 37, 1123-1128, 2006.

Zhou, F., Ciais, P., Hayashi, K., Galloway, J., Kim, D.-G., Yang, C., Li, S., Liu, B., Shang, Z., Gao, S.: Re-estimating $\mathrm{NH}_{3}$ emissions from Chinese cropland by a new nonlinear model, Environ. Sci. Technol., 50, 564-572, 2015. 